\title{
THE CLOSING LEMMA FOR GENERALIZED RECURRENCE IN THE PLANE
}

\author{
MARIA LÚCIA ALVARENGA PEIXOTO
}

\begin{abstract}
We prove a version of the Closing Lemma for $C^{r}$ vector fields on the plane, $r \geq 1$, and for a kind of recurrence obtained using the concept of prolongational limit sets. We call it generalized recurrence.

Given a nonperiodic point $p$ in the generalized recurrent set we perturb the vector field in order to get a new vector field arbitrarily close to it, with a closed orbit through $p$.
\end{abstract}

1. Introduction. This paper deals with the $C^{r}$ Closing Lemma, $r \geq 1$ on a noncompact manifold, the plane $\mathbf{R}^{2}$. Before we state precisely the hypothesis of the main theorem, we want to make some brief historical comments.

R. Thom, in 1960, was the first to consider the following problem. Can a vector field with a recurrent trajectory through a point $p$ be perturbed so as to obtain a new vector field with a closed trajectory through $p$ ? He claimed an affirmative answer, but his argument was valid only for a $C^{0}$-small perturbation. The perception that this problem is trivial in class $C^{0}$ and very difficult, $r \geq 1$, is due to M. M. Peixoto in $[\mathbf{1 2}]$.

In 1965, Charles Pugh proved the $C^{1}$ Closing Lemma for compact manifolds of dimension two and three. In 1967 he generalized it for arbitrary dimension and extended it to the case of closing a nonwandering trajectory, rather than a recurrent one [16]. In 1973, he proved that for a weaker type of recurrent point, for which $\alpha(p) \cap \omega(p) \neq \varnothing$, the $C^{2}$-double closing is not always possible on the 2-torus $T^{2}$ [17]. In 1983, C. Pugh and C. Robinson [18] established the Closing Lemma when $M$ is noncompact, provided the point $p$ lies on $\Omega_{c}=\{p \in \Omega: \alpha(p) \cup \omega(p) \neq \varnothing\}$.

It should be remarked that the $C^{r}$ Closing Lemma, $r \geq 1$, in the case that $M$ is the 2-torus $T^{2}$ and the vector field never vanishes, was proved in 1962 by M. M. Peixoto [12] and recently by C. Gutierrez in [4] for the so called constant type of vector fields on $T^{2}$ with finitely many singularities. In [5] C. Gutierrez gives a counterexample to the $C^{2}$ Closing Lemrra for the punctured torus. There is also "An Ergodic Closing Lemma" of R. Mañe (1982) that was used to characterize structurally stable diffeomorphisms of two-manifolds [6].

In the plane we do not have nontrivial recurrence, but there is a subtle kind of recurrence, the prolongational recurrence. So it makes sense to deal with the $C^{r}$ Closing Lemma in $\mathbf{R}^{2}$. A recent counterexample of C. Pugh in the punctured torus [19] disproves the $C^{1}$ Closing Lemma for prolongational recurrence on noncompact two-manifolds in general, but on compact ones it is an open question.

Received by the editors February 4, 1986 and, in revised form, January 5, 1987.

1980 Mathematics Subject Classification (1985 Revision). Primary 34Cxx; Secondary 34D30.

The author was partially supported by the CNPq-Brazil. 
In this paper, we consider the $C^{r}$ vector fields $X$ on $\mathbf{R}^{2}, r \geq 1$, which generate a flow $\varphi$, i.e., the trajectory through every point is defined for all time. The space $\mathscr{X}^{r}\left(\mathbf{R}^{2}\right)$ of these vector fields is endowed with the $C^{r}$ Whitney topology. That is the appropriate topology for the noncompact case (see [13 and 14]). In order to state the $C^{r}$ Closing Lemma on $\mathbf{R}^{2}$, we give some basic definitions.

(1.1) DEFInITION. For each $X \in \mathscr{X}^{r}\left(\mathbf{R}^{2}\right)$ and $x \in \mathbf{R}^{2}$, define the first positive (resp. negative) prolongational limit set by

$$
\left.J^{(\stackrel{+}{-})}(x)=\left\{y \in \mathbf{R}^{2}: \exists x_{n} \rightarrow x, t_{n} \rightarrow \infty \text { (resp. } t_{n} \rightarrow-\infty\right) \text { s.t. } \varphi_{t_{n}}\left(x_{n}\right) \rightarrow y\right\} .
$$

(1.2) Definition. For a subset $S \subset \mathbf{R}^{2}$, define

$$
J^{+}(S)=\bigcup_{x \in S} J^{+}(x) .
$$

(1.3) Definition. For each ordinal number $\alpha, X \in \mathscr{X}^{r}\left(\mathbf{R}^{2}\right)$ and $x \in \mathbf{R}^{2}$, call $J_{\alpha}(x)$ the prolongational limit set of order $\alpha$, defined by transfinite induction, as follows,

1. $J_{1}(x)=J^{+}(x)$.

2. Suppose that for all $\beta<\alpha, J_{\beta}(x)$ is defined.

(i) If $\alpha$ is a successor ordinal number, set

$$
J_{\alpha}(x)=J_{1}\left(J_{\alpha-1}(x)\right) .
$$

(ii) If $\alpha \geq \omega$ is a limit ordinal number, where $\omega$ is the first transfinite number, set

$$
J_{\alpha}(x)=\left\{y \in \mathbf{R}^{2}: \exists x_{n} \rightarrow x, y_{n} \rightarrow y \text { and ordinals } \beta_{n}<\alpha \text { with } y_{n} \in J_{\beta_{n}}\left(x_{n}\right)\right\} \text {. }
$$

It can be proved for any 2nd countable metric space that each prolongational limit set $J_{\alpha}(x)$ has a countable order $\alpha$.

(1.4) Definition. The generalized recurrent set $R(X)$ of $X$ (also called Auslander recurrent set $[\mathbf{1}]$ or prolongational recurrent set) is defined by

$$
R(X)=\left\{x \in \mathbf{R}^{2}: x \in J_{\alpha}(x) \text {, for some ordinal } \alpha\right\} .
$$

The nonwandering set $\Omega(X)$ is now the subset of $R(X)$ consisting of those points $x$ for which $x \in J_{1}(x)$. (Note that we have $x \in J_{1}^{+}(x) \Leftrightarrow x \in J_{1}^{-}(x)$, which implies that all of $\Omega(X)$ is included.)

(1.5) DEFINITION. A point $p \in R(X)$, which is not a periodic point is called prolongationally recurrent.

To clarify the concepts of generalized recurrent set and prolongationally recurrent point some examples are given below. In the example (a), $R(X)$ is the set of all prolongationally recurrent points $x$ plus the saddle node $y$. In (b) $R(X)$ is the whole closed shaded area. In (c) the three closed elliptic sectors constitute $R(X)$. In (d) we have an example in the sphere $S^{2}$ minus a point; with a sink in the other side of $S^{2} . R(X)$ is the set of the fixed saddles plus all separatrices that connect these saddles to infinity. All points of these separatrices are prolongationally recurrent. A similar example can be constructed for $x \in J_{\alpha}(x), \alpha$ being any countable ordinal number. 


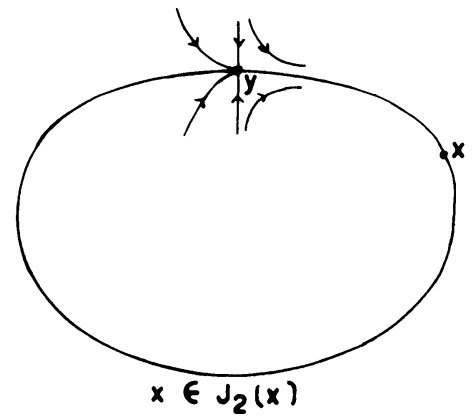

(a)

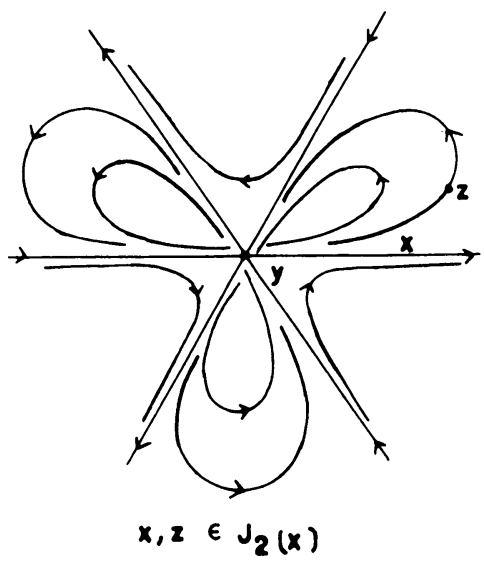

(c)

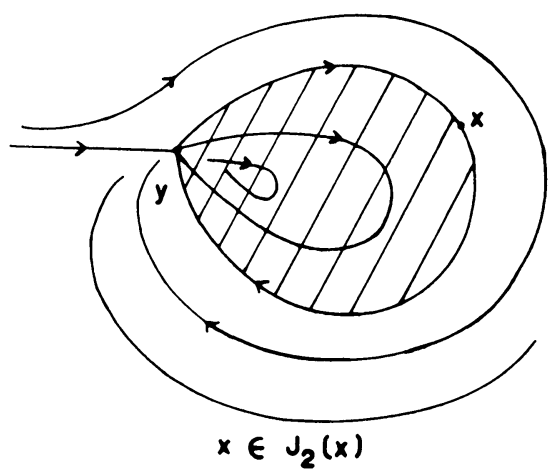

(b)

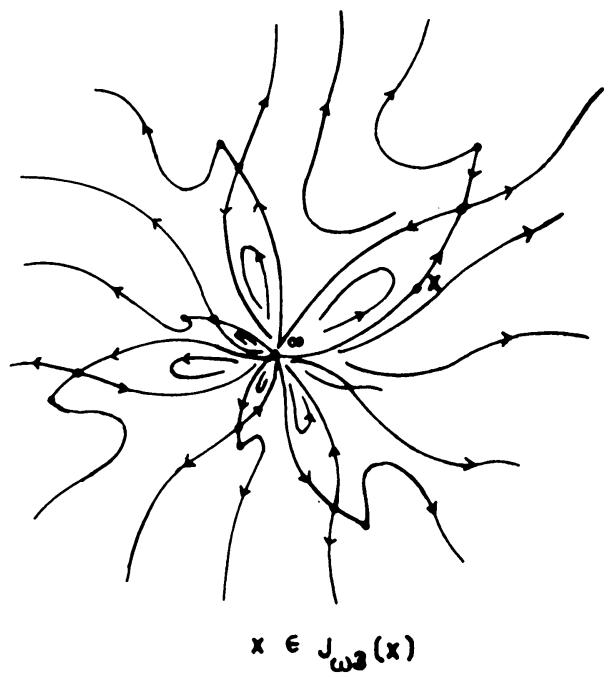

(d)

FIGURE (1.1)

(1.6) DEFinition. An $(\varepsilon, T)$-chain from $p$ to $q$ is a finite collection of trajectory arcs or segments $\left[x_{1}, y_{1}\right], \ldots,\left[x_{n}, y_{n}\right]$, each of duration $\geq T$ such that $d\left(y_{i}, x_{i+1}\right)<$ $\varepsilon, 0 \leq i \leq n$, where $p=y_{0}$ and $q=x_{n+1}$.

(1.7) Definition. A singularity $y$ of $X$ satisfies the shadow property if there exists a neighborhood $N$ of $y$ such that every $(\varepsilon, 1)$-chain in $N$ can be $\delta$-shadowed by a single trajectory arc of $X$ and $\delta \rightarrow 0$ uniformly as $\varepsilon \rightarrow 0$.

An $(\varepsilon, 1)$-chain is said to be $\delta$-shadowed by a single trajectory arc of $X$, if any point of the chain is less than $\delta$ distant from the trajectory arc and vice-versa. In [20] there is a description of all singularities which satisfy the shadow property, including the hyperbolic singularities. 


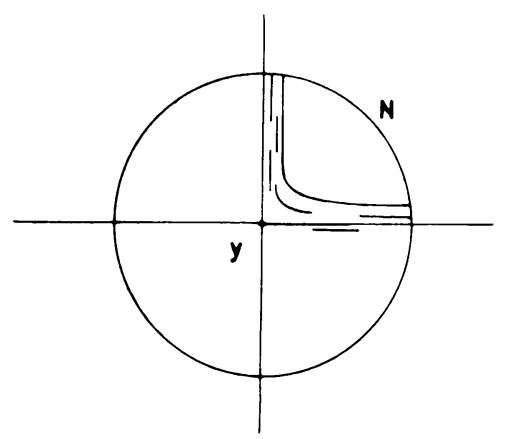

FIGURE (1.2)

(1.8) DEFinition A singularity $y$ of $X$ is semihyperbolic if it is isolated and the Jacobian $J(X)_{y}=0$, but its trace $\sigma(X)_{y} \neq 0$.

Semihyperbolic singularities are the saddle-node, the weak node or the weak saddle. It is possible to reduce their topological study by means of the restriction of $X$ to the center manifold $w_{X}^{c}$, which is an invariant curve tangent at $y$ to the null eigenvector of $D X_{y}$ (see [3]). In the case of the saddle-node, assume the saddlenode $y$ is $(0,0)$ and in local coordinates $\left(x_{1}, x_{2}\right)$ around $y$ the center manifold $W_{X}^{c}$ is the $x_{1}$-axis. If $X=f\left(x_{1}, x_{2}\right) \partial / \partial x_{1}+g\left(x_{1}, x_{2}\right) \partial / \partial x_{2}$, then the saddle-node is characterized by $g\left(x_{1}, x_{2}\right)=x_{2} h\left(x_{1}, x_{2}\right)$, with $h\left(x_{1}, x_{2}\right)<0$ and $f\left(x_{1}, 0\right)>0$ or $f\left(x_{1}, 0\right)<0$ for $x_{1} \neq 0$.

We now state the theorem which is the object of this paper.

(1.9) THEOREM (CLOSING LEMMA). Let $p$ be a prolongationally recurrent point of $X \in \mathscr{X}^{r}\left(\mathbf{R}^{2}\right), r \geq 1$, with singularities which are either semihyperbolic or satisfy the shadow property. Let $\mathscr{U}$ be a neighborhood of $X$ in $\mathscr{X}^{r}\left(\mathbf{R}^{2}\right)$. Then there exists $Y \in \mathscr{U}$ such that $Y$ has a closed orbit through $p$.

It might be ciesirable to have such a Closing Lemma when the singularities are isolated and not too complicated. This does not seem to be an easy matter. In example (b) of Figure (1.1) the singularity does not satisfy our restriction and we do not know if we can get a closed orbit through $x \in R(X)$. In example (c) of Figure (1.1) the singularity also does not satisfy our requirements. Still the same method used in the proof of our theorem allows us to get a closed orbit through $x \in R(X)$. But through $z \in R(X)$, or through any point such that the corresponding $\alpha$ and $\omega$-limit sets are the singularity $y$, this does not happen.

In $\S 2$ we show that if $X$ is devoid of singularities on $\mathbf{R}^{2}$, the Closing Lemma is trivially true because $R(X)$ is empty. In $\S \S 3$ and 4 we give a number of concepts and results necessary for our proof, of which the box-chain lemma is the central result. This lemma shows that when $y \in J_{\alpha}(x), \alpha$ any ordinal, then there exists between $x$ and $y$ a chain of flowboxes and trajectory arcs connecting their central portions. The idea of the proof of our Closing Lemma is to consider a box-chain loop through a prolongationally recurrent point $p \in R(X)$. Next we perturb the vector field $X$ locally in each flowbox in order to connect the two trajectory arcs of the chain that arrive and leave the flowbox. Then we come to the final step where the restriction on the nature of the singularities comes in. The semihyperbolic singularities existing in the chain can be eliminated through a perturbation, and 
the ones which satisfy the shadow property can be bypassed. Thus we get our closed orbit.

We remark that in the plane the Closing Lemma on $\Omega(X)$ is true and trivial without any restriction on the nature of the singularities.

2. The nonsingular case. A vector field with no nonwandering points is not necessarily devoid of recurrence phenomena. The example in the cylinder $S^{1} \times \mathbf{R}$ indicated in Figure (2.1) has a subtle kind of cycle, which belongs to the generalized recurrent set $R(X)$. Nevertheless on the plane, this fact does not occur. Actually we have the following.

(2.1) THEOREM. Let $X$ be a $C^{r}$ vector field on $\mathbf{R}^{2}$. If $\Omega(X)=\varnothing$, then $R(X)=\varnothing$.

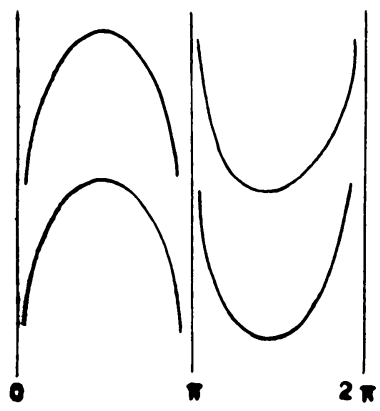

FigURE (2.1)

The proof of this theorem is an easy consequence of a theorem of Nitecki [9] and the two easy lemmas below. First we give a definition.

(2.2) Definition. Let $\varphi$ be the $X$-flow. We call $\varphi C^{r}$ nonexplosive, $r \geq 0$, if $\Omega(\varphi)=\varnothing$ and there exists a neighborhood $\mathscr{U}$ of $\varphi$ in the Whitney $C^{r}$ topology such that any flow $\psi \in \mathscr{U}$ has $\Omega(\psi)=\varnothing$.

The theorem of Nitecki gives several conditions equivalent to the property that a $X$-flow $\varphi$ (on a noncompact manifold) and its $C^{0}$ perturbations have only wandering points, i.e., $\varphi$ is $C^{0}$ nonexplosive. The equivalence we use here is

(2.3) $\varphi$ is $C^{0}$ nonexplosive $\Leftrightarrow$ the generalized recurrent set $R(\varphi)$ is empty.

(2.4) LEMMA. Let $M$ be a noncompact (resp. compact) manifold and $\mathscr{X}_{0}^{r}(M)$ the set of all $C^{r}$ nonsingular vector fields on $M, r \geq 0$. Then $\mathscr{X}_{0}^{r}(M)$ is an open set in $\mathscr{Q}^{r}(M)$.

ProOF. Consider $X_{0} \in \mathscr{X}_{0}^{r}(M)$, so that $X_{0}$ is a section in the tangent bundle of $M$ that does not intersect the 0 -section. Then we can find a neighborhood $N$ of the section $X_{0}$ in the Whitney topology (resp. topology of uniform convergence) such that any $Y \in N$ is nowhere zero, i.e., $Y \in \mathscr{X}_{0}^{r}(M)$. Thus $\mathscr{X}_{0}^{r}(M)$ is open in $\mathscr{X}^{r}(M)$.

(2.5) LEMMA. $X$ is nonsingular on $\mathbf{R}^{2} \Leftrightarrow \Omega(X)=\varnothing$.

Proof. Suppose $\Omega(X) \neq \varnothing$. Let $p \in \Omega(X)$ and $\Sigma$ be a transversal segment at $p$. Then for any neighborhood $V$ of $p$, there exist $x, y \in V \cap \Sigma$ such that $y=\varphi_{t}(x)$ 
for some $t>0$. Suppose $y$ is the first point on the semitrajectory $\gamma_{t}(x)$ belonging to $\Sigma$. Consider the jordan curve $L$ composed by the trajectory arc $x y$ and the segment $[x, y] \subset \Sigma$. By the Poincaré-Bendixson theorem, in the interior of $L$ there exists a singularity, which is a contradiction. Thus $\Omega(X)=\varnothing$.

Conversely, if $\Omega(X)=\varnothing$, it is obvious that $X$ is nonsingular, and the lemma is proved.

Proof of TheOREM (2.1). Since $X$ is a $C^{r}$ vector field on $\mathbf{R}^{2}$, it follows from Lemmas (2.4) and (2.5) that if $\Omega(X)=\varnothing$ then the $X$-flow $\varphi$ is $C^{r}$ nonexplosive for any $r \geq 0$. In this case the theorem of Nitecki (2.3) implies that $R(X)=\varnothing$.

REMARK. Mendes proves in [7] the analogue of Theorem (2.1) for diffeomorphisms of $\mathbf{R}^{2}$. We can prove the flow case from the Mendes theorem for the time-one diffeomorphism $\varphi_{1}$ of the $X$-flow, but this is not immediate.

3. Flowbox system. Let $X$ be a $C^{r}$-vector field on a noncompact manifold $M^{n}, n \geq 2$ endowed with a Riemannian metric. We denote the set of regular points of $X$ by $M_{0} \subset M^{n}$, and the $X$-flow by $\varphi$.

Introduce a continuous system of flowboxes, associating a flowbox $N_{x}$ to each point $x \in M_{0}$ in a continuous way. We need in $\S 4$ the fact that if two flowboxes $N_{x}$ and $N_{y}$ of the system intersect each other, their union contains a common transversal, that is, a segment transversal to $X$, which crosses all trajectories of $X$ in $N_{x}$ and in $N_{y}$. For this we define on an orientable manifold $M^{2}$ what we call the orthogonal system of flowboxes.

Consider $T_{x} M_{0}=\operatorname{Span}(X) \oplus \operatorname{Span}\left(X^{\perp}\right)$, where $X^{\perp}$ is a vector field on $M^{2}$ orthogonal to $X$ with flow $\varphi^{\perp}$. For any $x \in M_{0}$, there is a $\delta>0$ and a neighborhood $V$ of $x, V \subset M_{0}$ such that for all $\left|t_{0}\right|<\delta,\left|\tau_{0}\right|<\delta$ the intersection of the trajectory $\operatorname{arcs}\left\{\varphi_{t}\left(\varphi_{\tau_{0}}^{\perp}(x)\right)\right\}$ and $\left\{\varphi_{\tau}^{\perp}\left(\varphi_{t_{0}}(x)\right)\right\}$ is a unique point in $V$, and the map $f_{x, \delta}:[-\delta, \delta] \times[-\delta, \delta] \rightarrow M_{0}$, defined by $f_{x, \delta}\left(t_{0}, \tau_{0}\right)=\left\{\varphi_{t}\left(\varphi_{\tau_{0}}^{\perp}(x)\right)\right\} \cap\left\{\varphi_{\tau}^{\perp}\left(\varphi t_{0}(x)\right)\right\}$ is a $C^{r}$-embedding. Since $M^{2}$ is locally compact we may define a positive continuous function $\delta(x)$ on $M_{0}$, such that $f_{x, \delta(x)}$ is an embedding for each $x \in M_{0}$.

(3.1) DEFINITION. An orthogonal system of flowboxes is the set $\mathscr{F}=\left\{f_{x, \delta(x)}\right\}$, where $\delta(x)$ is a positive continuous function on $M_{0}$, chosen as above.

(3.2) DEFinition. The flowbox of radius $\delta(x)$ at $x \in M_{0}$ is the image $N_{x}$ of the map $f_{x, \delta(x)}$.

(3.3) DEFINITION. If $0<c<1$, the $c$-central portion of the interval $[-\delta, \delta]$ is the interval $[-c \delta, c \delta]$ and the $c$-central portion of $N_{x}$ is the set

$$
\left\{\left\{\varphi_{t}\left(\varphi_{\tau_{0}}^{\perp}(x)\right)\right\} \cap\left\{\varphi_{\tau}^{\perp}\left(\varphi_{t_{0}}(x)\right)\right\}:\left|t_{0}\right| \leq \delta(x),\left|\tau_{0}\right| \leq c \delta(x)\right\} .
$$

For more general situations below, we define a canonical system of flowboxes in a manifold $M$ of arbitrary dimension as follows. For each $x \in M_{0}$, there exists a $\delta>0$ giving the $C^{r}$-embedding $f_{x, \delta}:[-\delta, \delta] \times \pi_{x}(\delta) \rightarrow M_{0}$, defined by $f_{x, \delta}(t, v)=$ $\varphi_{t}\left(\exp _{x}(v)\right)$, where $\pi_{x}(\delta)$ is the disc of radius $\delta$ in the hyperplane complementary to $X$ in $T_{x} M_{0}$. As noted above, we may choose a positive function $\delta$ on $M_{0}$ so that $f_{x, \delta(x)}$ is an embedding for each $x \in M_{0}$.

(3.4) DEFINITION. Call $\mathscr{F}=\left\{f_{x, \delta}\right\}$ a canonical system of flowboxes. Just as in the orthogonal system of flowboxes, we define the flowboxes $N_{x}$ of radius $\delta(x)$ at $x \in M_{0}$ and the $c$-central portion of $N_{x}$ for a canonical system of flowboxes.

By (3.1) and (3.2), if two flowboxes $N_{x}$ and $N_{y}$ in an orthogonal system of flowboxes intersect each other, their union has a common transversal, indeed a 
trajectory arc of the vector field $X^{\perp}$. This does not necessarily happen for a canonical system of flowboxes.

Fix an orthogonal system of flowboxes on $M_{0} \subset M^{2}$.

(3.5) Lift Lemma. Given an $\varepsilon(x)$-neighborhood $\mathscr{U}$ of $X \in x^{r}\left(M^{2}\right), r \geq 1$, there exists a continuous function $0<c(x)<1$ on $M_{0}$ such that any two points $p, q$ in opposite transversal edges of the $c(x)$-central portion of the flowbox $N_{x}$ around $x$ can be connected by a trajectory arc of a vector field $Y \in \mathscr{U}$, with $Y \equiv X$ outside $N_{x}$.

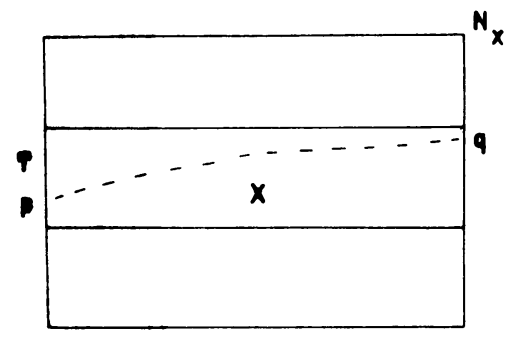

FiguRE (3.1)

ProOF. By (3.1) and (3.2), each flowbox $N_{x}$ has radius $\delta(x)$, where $\delta(x)$ is a positive continuous function on $M_{0}$. We can take $\delta(x)<1$. For $0 \leq u \leq 1$, define the vector field $X(u)$ on $M^{2}$ by $X(u)=X+(\varepsilon / B) u \beta Z$, where $\varepsilon \leq \inf _{z \in N_{x}} \varepsilon(z), \beta$ is a bump function such that $0<\beta \leq 1$ inside $N_{x}$ and $\beta \equiv 0$ outside $N_{x}, Z=X^{\perp}$ normalized and $B=\max _{0 \leq j \leq r} \sup \left\{\left\|\beta^{(j)}(z)\right\| ; z \in N_{x}\right\}$. It follows that $X(u) \in \mathscr{U}$ and $X(u) \equiv X$ outside $N_{x}$.

We use the idea of a "minimum lift" from [12], as follows. Consider a compact central portion of $N_{x}$ and let $\tau$ denote the segment determined by this central portion on the left transversal edge of $N_{x}$. (See Figure (3.1).) For every point $y \in \tau$, consider the trajectories of $X(0)$ and $X(1)$ through $y$ and call $l(y)>0$ the length of the segment determined by them on the right transversal edge of $N_{x}$. Since $l(y)$ is continuous in $y$ and $\tau$ is compact, there is a minimum lift $l>0$ such that $l(y) \geq l$ for all $y \in \tau$. Clearly if we diminish $\tau$, the minimum lift increases. Thus we can choose $c, 0<c<1$, so small that the minimum lift corresponding to the $c$-central portion of $N_{x}$ is greater than the radius $c \delta$ of this central portion. Thus if $p$ and $q$ are points in opposite transversal edges of the $c$-central portion of $N_{x}$, there exists a vector field $Y=X\left(u_{0}\right)$ for some $0 \leq u_{0} \leq 1$, connecting $p$ to $q$ by a trajectory are through $N_{x}$ and $Y \in \mathscr{U}(X)$. By the continuity of the orthogonal system of flowboxes we can choose the function $c$ continuous in $x$, and the lemma is proved.

4. Box-chains. Let $X \in \mathscr{X}^{r}(M)$ be a vector field with isolated singularities. Because some results are valid in any dimension, we begin in $M^{n}$, a manifold of arbitrary dimension. Fix a canonical covering of $M_{0} \subset M$ by flowboxes of radius $\delta(x)$, as in (3.4).

(4.1) DEFINITION. A box-chain from $x$ to $y$ is a finite and alternate sequence of flowboxes $N_{i}$ and curves $\gamma_{i}$, such that $\gamma_{i}$ is either a trajectory arc or two trajectory arcs with a singularity between them and $\gamma_{i}$ joins $N_{i}$ to $N_{i+1}, i=1,2, \ldots, n-1$. 


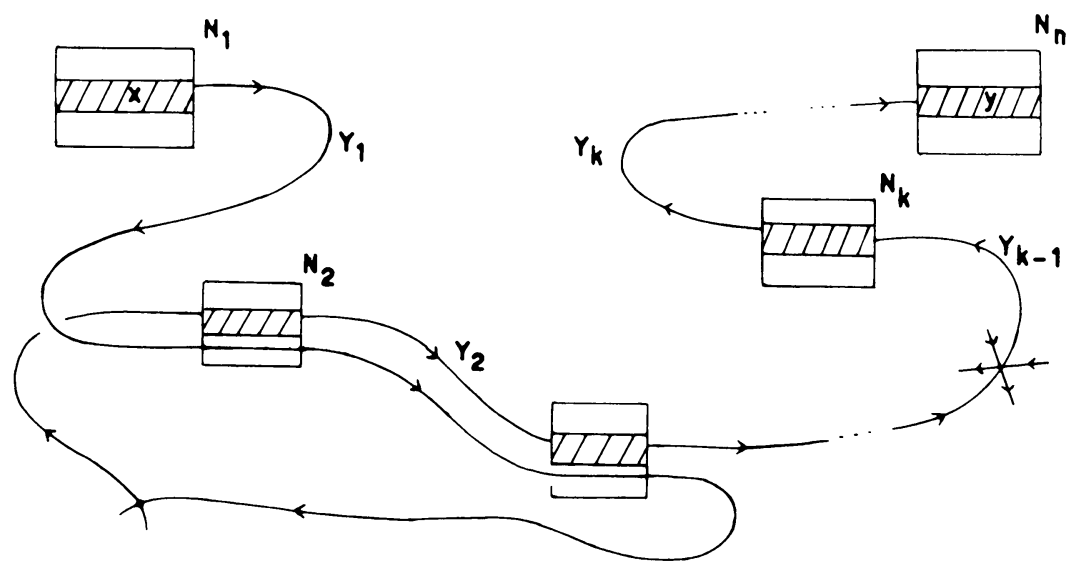

FIGURE (4.1)

(4.2) DEFINITION. If $c=c(x)$ is a positive function on $M_{0}$, the box-chain is $c$-central if $\gamma_{i}$ joins the $c$-central portion of $N_{i}$ to that of $N_{i+1}$. See Figure (4.1).

(4.3) BOX-CHAIN Lemma. Let $y \in J_{\alpha}(x)$, where $\alpha$ is any ordinal number and $x, y$ are any points in $M_{0}$. Assume the $X$-flow has only isolated singularities. Then, for every continuous function $c=c(x)$ on $M_{0}, 0<c<1$, there exists a $c$-central box-chain from $x$ to $y$.

PROOF. We prove this lemma by transfinite induction on $\alpha$.

STEP 1. Take $\alpha=k>0$, an integer, so

$$
y \in J_{k}(x)=\underbrace{J_{1}\left(J_{1} \cdots\left(J_{1}(x)\right)\right.}_{k \text {-times }} \cdots) .
$$

This implies we can choose points $p_{0}, p_{1}, \ldots, p_{m}, m \geq k$, with $p_{0}=x$ and $p_{m}=y$ such that $p_{i+1} \in J_{1}\left(p_{i}\right)$ and some $p_{i}$ may be singularities. We have $m \geq k$ because we assume, without loss of generality, that for each singularity $p_{j}$ we choose regular points $p_{j-1}$ and $p_{j+1}$ in one of its $\alpha$-separatrix and $\omega$-separatrix respectively such that $p_{j-1} \in J_{1}\left(p_{j-2}\right)$ and $p_{j+2} \in J_{1}\left(p_{j+1}\right)$.

Let $z_{0}=p_{0}, z_{1}, \ldots, z_{n}=y$ be the regular points $p_{i}$. In the canonical system of flowboxes, there exist flowboxes $N_{i}$ around each $z_{i}, i=1, \ldots, n$. We can have $z_{i+1} \in J_{1}\left(z_{i}\right)$ or $p_{j} \in J_{1}\left(z_{i}\right)$ and $z_{i+1} \in J_{1}\left(p_{j}\right), p_{j}$ a singularity. In any case there exist sequences $z_{i}^{n} \rightarrow z_{i}$ and $t_{n}^{i} \rightarrow \infty$ such that $\varphi_{t_{n}^{i}}\left(z_{i}^{n}\right) \rightarrow z_{i+1}$ or $\varphi_{t_{n}^{i}}\left(z_{i}^{n}\right) \rightarrow p_{j}$. So for each $i$, there exists a curve $\gamma_{i}$ which is either a trajectory arc of $\mathscr{O}_{+}\left(z_{i}^{n}\right)$ for some $n$ or two trajectory arcs of $\mathscr{O}_{+}\left(z_{i}\right)$ and $\mathscr{O}_{-}\left(z_{i+1}\right)$ with a singularity $p_{j}$ at their intersection connecting the central portions of $N_{i}$ and $N_{i+1}$. In the first case we can choose $n$ large enough so $\gamma_{i}$ joins the $c$-central portion of $N_{i}$ to that of $N_{i+1}$, for any $0<c<1$, by the definition of prolongational limit set. In the second case, $\gamma_{i}$ always leaves $N_{i}$ and arrives at $N_{i+1}$ in their $c$-central portion, for any $c$, since $\gamma_{i}$ is composed of trajectory arcs through $z_{i}$ and $z_{i+1}$. Then we have a $c$-central box-chain from $x$ to $y$.

STEP 2. Take $\alpha$ to be any ordinal number. 
We suppose the lemma is not true for a subset $S$ of the ordinal numbers. From the well-ordering of $S$, there exists a smallest element $\bar{\alpha}$. Since the lemma is true for all $\beta<\bar{\alpha}$, let us prove it is true as well for $\bar{\alpha}$, which is absurd. We have two cases

(a) Suppose $\bar{\alpha}$ is a limit ordinal number. By Definition (1.3),

$$
J_{\bar{\alpha}}(x)=\left\{y \mid \exists x_{n} \rightarrow x, y_{n} \rightarrow y \text { such that } y_{n} \in J_{\beta_{n}}\left(x_{n}\right), \text { where } \beta_{n}<\bar{\alpha}\right\} .
$$

Since $\beta_{n}<\bar{\alpha}$, by the induction hypothesis the lemma is true for $y_{n} \in J_{\beta_{n}}\left(x_{n}\right)$, i.e., there exists a $c$-central box-chain from $x_{n}$ to $y_{n}$ for any $0<c<1$. Thus we can choose $n$ so large that $x_{n}$ and $y_{n}$ are in the $c$-central portion of $N_{x}$ and $N_{y}$ respectively and the $c / 2$-central portion of $N_{x_{n}}$ and $N_{y_{n}}$ are in the $c$-central portion of $N_{x}$ and $N_{y}$ respectively. This implies that the trajectory arc $\gamma_{0}$ leaves the $c$-central portion of $N_{x}$ and $\gamma_{n-1}$ arrives at that of $N_{y}$. Thus we have a $c$-central box-chain from $x$ to $y$, where $y \in J_{\bar{\alpha}}(x)$, which is a contradiction.

(b) Suppose $\bar{\alpha}$ is not a limit ordinal number. We have $y \in J_{\bar{\alpha}}(x)=J_{1}\left(J_{\bar{\alpha}-1}(x)\right)$. This implies that there exists $y^{\prime} \in J_{\bar{\alpha}-1}(y)$ such that $y \in J_{1}\left(y^{\prime}\right)$. Since $\bar{\alpha}-1<\bar{\alpha}$, by the induction hypothesis there exists a $c$-central box-chain from $x$ to $y^{\prime}$, for any $0<c<1$. As $y \in J_{1}\left(y^{\prime}\right)$, by definition we can choose a trajectory arc $\gamma_{n}$ leaving the $c$-central portion of $N_{y^{\prime}}$ and arriving at that of $N_{y}$, for any $0<c<1$. Then, adding the trajectory arc $\gamma_{n}$ and the flowbox $N_{y}$ to the box-chain from $x$ to $y^{\prime}$, obtained above, we get a $c$-central box-chain from $x$ to $y$, for any $0<c<1$, which is a contradiction. Thus (4.3) is true for all ordinal numbers $\alpha$ and $x, y \in M_{0}$, such that $y \in J_{\alpha}(x)$.

(4.4) THEOREM. Let $X$ be a vector field on $M$ as in (4.3) and $x \in M_{0}$ be prolongationally recurrent, i.e., $x \in J_{\alpha}(x)$ for some ordinal $\alpha$. Then for every continuous function $c$ and $M_{0}, 0<c<1$, there exists a c-central box-chain loop from $x$ to $x$.

PROOF. It follows immediately from the box-chain lemma, where we consider $y=x$.

(4.5) BOX-CHAIN SIMPLE IN $\mathbf{R}^{2}$. From the preceding theorem we get a $c$ central box-chain from $x$ to $x$, where $x$ is a prolongationally recurrent point. But that chain might cross some of the flowboxes twice or more times, producing loops, and it might have flowboxes intersecting each other. In order to get a closed orbit from a $c$-central box-chain, we show here that in the plane we can make it box-chain simple, in the sense that the curve $\gamma_{i}$ crosses $N_{i}$ just once, leaving $N_{i}$ and arriving at $N_{i+1}$, without crossing any $N_{j}, j \neq i, i+1$ and that the flowboxes $N_{i}$ are disjoint. Here we consider the orthogonal system flowboxes as in (3.1). Using the theorem (4.4), consider a closed continuous curve $\Gamma$ through the point $x$, composed by arcs so that

$$
\Gamma=x, \hat{\tau}_{0}, \gamma_{0}, \tau_{1}, \gamma_{1}, \tau_{2}, \ldots, \gamma_{n-2}, \tau_{n-1}, \gamma_{n-1}, \hat{\tau}_{n}, x
$$

where $\gamma_{0}, \gamma_{1}, \ldots, \gamma_{n-1}$ are the trajectory arcs given by the box-chain and $\tau_{i}$ is a transversal segment inside the flowbox $N_{i}$ connecting the curve $\gamma_{i-1}$ to $\gamma_{i}$ while $\hat{\tau}_{0}, \hat{\tau}_{n}$ are the two parts of the transversal segment, which contain $x$, as shown in Figure (4.2). Here $\Gamma$ can cross itself only in the transversal segments $\tau_{i}$, or in the singularities since the curves $\gamma_{i}$ are trajectory arcs. Thus let us choose first a simplified oriented loop $\Gamma^{\prime}$ from $x$ to $x$, i.e., each $\gamma_{i}$ in $\Gamma^{\prime}$ crosses $N_{i}$ and $N_{i+1}$ just 
once and $\gamma_{i}$ does not cross any $N_{j}, j \neq i, i+1$. We do that choosing the most future arc, when $\Gamma$ crosses itself, as in Figure (4.3). Roughly speaking, we call $\Gamma^{\prime}$ a box-chain loop through $x$, simplified through the construction of the oriented loop $\Gamma^{\prime}$ above.

We know that a curve crosses a transversal segment $\tau$ just once in the topological sense if it crosses the transversal segment as in Figure 4.4.

(4.6) LEMMA. A simplified box-chain loop in $\mathbf{R}^{2}$ crosses a transversal segment at most once in the topological sense.

PrOOF. Suppose a simplified box-chain loop $\Gamma^{\prime}$ crosses a transversal segment $\tau$ twice. We obtain a jordan curve $L$ composed by the part of $\Gamma^{\prime}$ between the two crossings plus the transversal segment connecting them. (See Figure 4.5.)

By the Jordan curve theorem, $\Gamma^{\prime}$ enters in a disc $D$ limited by $L$ and cannot leave it, since $\Gamma^{\prime}$ does not cross itself $\left(\Gamma^{\prime}\right.$ is simplified) and cannot cross the transversal segment $\tau$ in the opposite direction. This is absurd, since $\Gamma^{\prime}$ is a loop.

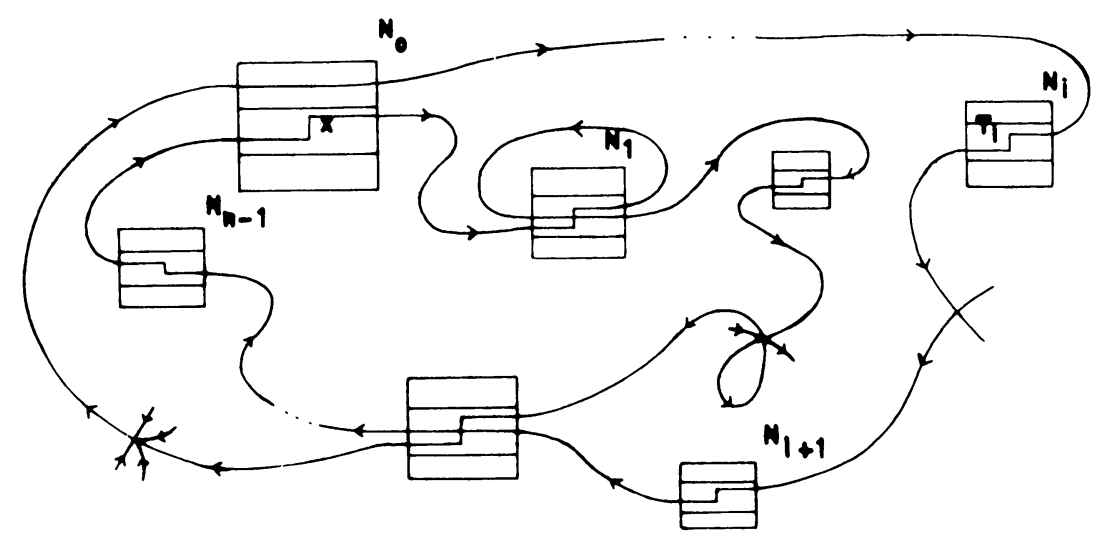

FiguRE (4.2)

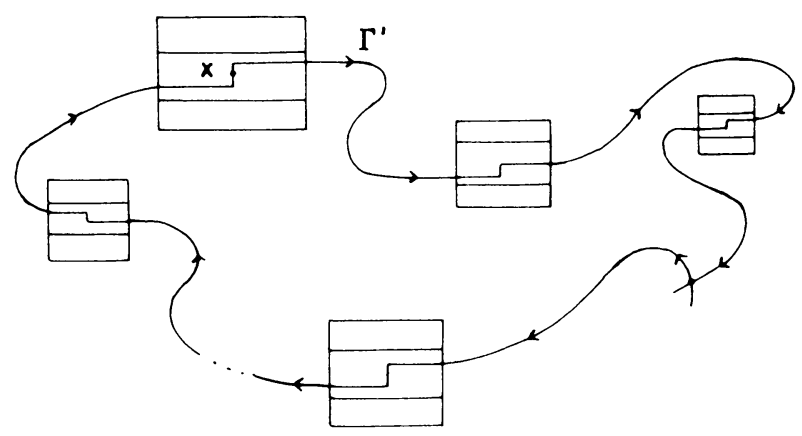

FiguRE (4.3) 

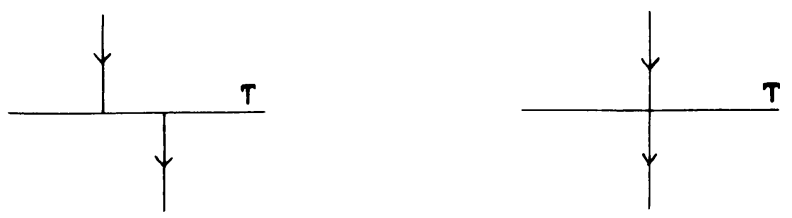

FIGURE (4.4)

(4.7) Lemma. Any simplified box-chain loop $\Gamma^{\prime}$ in $\mathbf{R}^{2}$ has these properties.

(a) No loop arc $\gamma_{i}$ meets a flowbox $N_{j}, j \neq i, i+1$.

(b) The flowboxes of the box-chain $\Gamma^{\prime}$ are disjoint.

ProOF. (a) In fact, since the arc $\gamma_{j-1} \gamma_{j}$ already crosses the transversal segment $\tau_{j}$ through $z_{j} \in N_{j}$, by the Lemma (4.6) it is not possible to have $\gamma_{i}$ crossing $N_{j}$, for any $j \neq i, i+1$.

(b) Suppose $N_{i} \cap N_{j} \neq \varnothing, i \neq j$. From the orthogonal system of flowbox, any flowbox around a regular point has transversal segments that are trajectory arcs of the orthogonal vector field $X^{\perp}$. Thus $N_{i} \cup N_{j}$ has a common transversal segment $\tau$, as seen in Figure 4.6. By Lemma (4.6), intersection is impossible, or $\Gamma^{\prime}$ would cross the transversal segment twice. So $N_{i} \cap N_{j}=\varnothing$ for any $i, j, i \neq j$.

By Lemma (4.7), the box-chain loop $\Gamma^{\prime}$ is in fact simple, and by construction it is even more central with respect to its flowboxes than $\Gamma$, and the flowboxes in $\Gamma^{\prime}$ might be less in number than the ones in $\Gamma$. See Figures (4.2) and (4.3).

REMARK $1 . \Gamma^{\prime}$ might contain some singularities, but if we assume $X$ has only hyperbolic singularities (in this case saddles), then we can get a nonsingular $c$ central chain-box loop directly from the box-chain lemma. In fact, in the box-chain lemma proof, when constructing the box-chain, we can avoid taking singularities in the choice of the points prolongationally related. This is possible because the singularities are hyperbolic. Thus we can always choose in $J_{1}\left(z_{i}\right)$, for some point $z_{i}$ in the $\alpha$-separatrix of a singularity $p_{j}$, a regular point in the $\omega$-separatrix of this singularity, instead of the singularity itself. We cannot always do this when the singularities are isolated as in Figure (4.7)(b), where we cannot avoid $p_{j}$.

We need two more steps to obtain a closed orbit through $x \in R(X)$ proving Closing Lemma (1.9). The first step is to prove the Singular Closing Lemma, which gives a closed curve through $x$, composed of trajectory arcs and singularities between them. The second step is to perturb the vector field obtained to eliminate

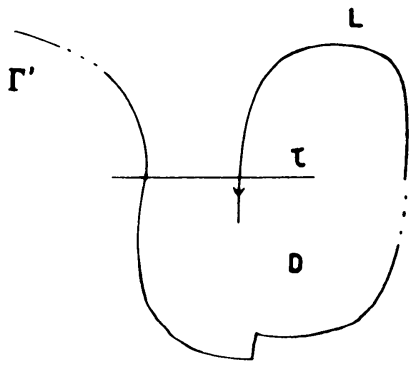

FIGURE (4.5) 


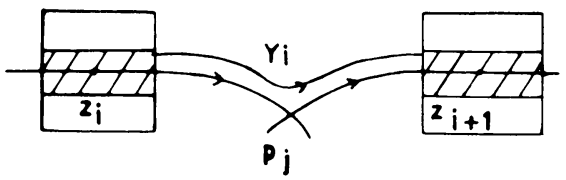

(a)

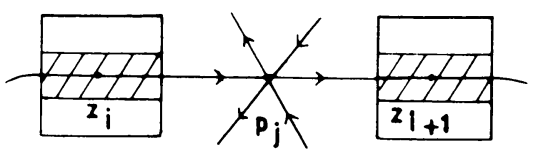

(b)

FigURE (4.6)
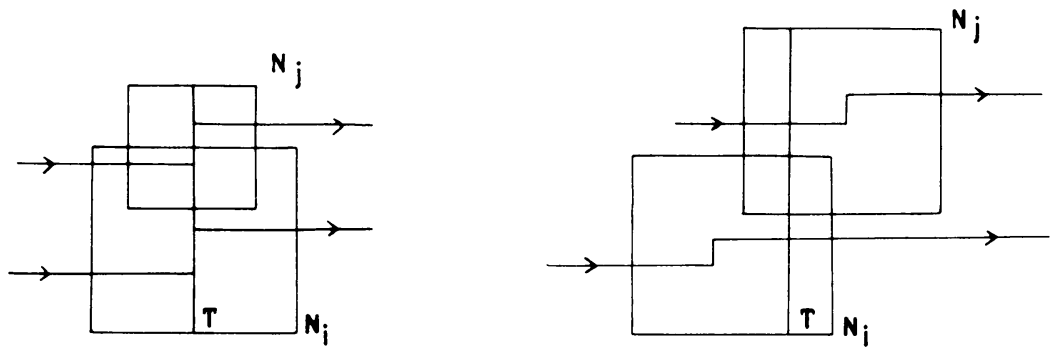

FIGURE (4.7)

the singularities of the closed curve, getting a closed orbit through $x$. We need first a simple lemma.

(4.8) Lemma. Suppose $X \in \mathscr{Z}^{r}\left(\mathbf{R}^{2}\right), r \geq 1$ and $\mathscr{U}$ is a $\varepsilon(x)$-neighborhood of $X$ in $\mathscr{X}^{r}\left(\mathbf{R}^{2}\right)$. If $\Delta_{1}, \Delta_{2}, \ldots$ in $\mathscr{X}^{r}\left(\mathbf{R}^{2}\right)$ have disjoint supports and $Y_{i}=X+\Delta_{i}$ is in $\mathscr{U}$ for each $i$, then $Y=X+\sum_{i} \Delta_{i}$ is also in $\mathscr{U}$.

Proof. The $\varepsilon(x)$-neighborhood $\mathscr{U}$ of $X$ in the Whitney topology is defined pointwise by

$$
\mathscr{U}(X, \varepsilon(x))=\left\{Y \in \mathscr{X}^{r}\left(\mathbf{R}^{2}\right):\|Y(x)-X(x)\|_{r}<\varepsilon(x) \text { for all } x \in \mathbf{R}^{2}\right\},
$$

where

$$
\|Y(x)-X(x)\|_{r}=|Y(x)-X(x)|+\left\|D^{1}(Y-X)(x)\right\|+\cdots+\left\|D^{r}(Y-X)(x)\right\| .
$$

Since each $Y_{i}=X+\Delta_{i} \in \mathscr{U}$ and $\Delta_{1}, \Delta_{2}, \ldots$ have disjoint supports, then

$$
\begin{aligned}
& \|Y(x)-X(x)\|_{r}=\left\|\sum_{i} \Delta_{i}\right\|_{r} \\
& \quad=\left|\Delta_{1}(x)+\Delta_{2}(x)+\cdots\right|+\cdots+\left\|D^{r}\left(\Delta_{1}\right)(x)+D^{r}\left(\Delta_{2}\right)(x)+\cdots\right\| \leq \varepsilon(x),
\end{aligned}
$$

which implies $Y \in \mathscr{U}$.

(4.9) Singular Closing Lemma. Suppose $X \in \mathscr{X}^{r}\left(\mathbf{R}^{2}\right)$ with all singularities isolated, $p \in R(X)$ a prolongationally recurrent point and $\mathscr{U}$ a neighborhood of $X$ in $\mathscr{X}^{r}\left(\mathbf{R}^{2}\right)$. Then there exists a vector field $Z \in \mathscr{U}$ with a closed curve through $p$, composed of trajectory arcs and singularities of $Z$.

Proof. By (4.4) and (4.5), for every continuous function $0<c<1$ on $\mathbf{R}_{0}^{2} \subset \mathbf{R}^{2}$, $\mathbf{R}_{0}^{2}$ being the set of regular points of $X$, there exists a $c$-central box chain loop $\Gamma^{\prime}$ from $p$ to itself, which is box simple. Consider a smaller neighborhood $\mathscr{U}^{\prime}$ of $X$, $\mathscr{U}^{\prime} \subset \mathscr{U}$. By (3.5) there is a positive continuous function $c<1$ on $\mathbf{R}_{0}^{2}$ such that 
any two points in opposite transversal edges of a $c$-central portion of a flowbox $N_{x}$ can be connected by a $X^{\prime}$-trajectory across $N_{x}$, where $X^{\prime} \in \mathscr{U}^{\prime}$ is such that $\operatorname{supp}\left(X^{\prime}-X\right) \subset N_{x}$. Consider the flowboxes $N_{i}, i=1, \ldots, n$, of the box-chain and make this connection construction in each $N_{i}$ for the curves $\gamma_{i-1}$ and $\gamma_{i}$, that arrive and leave $N_{i}$ respectively in opposite transversal edges of the $c$-central portion of $N_{i}$. Thus we get $Y_{1}, Y_{2}, \ldots, Y_{n} \in \mathscr{U}^{\prime}$. By Lemma (4.8), $Y=X=\sum_{i=1}^{\infty}\left(Y_{i}-X\right)$ also belongs to $\mathscr{U}^{\prime}$. By construction, $Y$ has a closed curve $\gamma$ composed only of trajectory arcs and singularities between them, which may not contain $p$ but passes near $p$. Let $q$ be a point of $\lambda$ close to $p$. We consider a local translation $f: \mathbf{R}^{2} \rightarrow \mathbf{R}^{2}$ that takes $q$ to $p$, which is a $C^{\infty}$ diffeomorphism near the identity in a small neighborhood $V$ of $p$ and $q$ and equal to the identity outside a neighborhood containing $\bar{V}$. This $f$ induces a map $f_{*}$ (see [11]) such that the vector field $Z=f_{*} Y=d f \circ Y \circ f^{-1}$ has a closed curve $\bar{\lambda}$ through $p$, which is a translation of $\lambda$ and $z \in \mathscr{U}$. Then (4.9) is proved.

REMARK 2. Although it is intuitive that such a local translation $f$ exists, we give a construction. Consider a $C^{\infty}$ function $\psi$ on $\mathbf{R}^{2}$ such that $\operatorname{supp} \psi=D_{1}^{2}$ and $\psi=1$ on $D_{1 / 2}^{2}$. Consider the vector field $W=W(z ; q, v, \mu)=\psi((z-q) / \mu) v$ on $\mathbf{R}^{2}$ with $z, q, v \in \mathbf{R}^{2}, q$ given above, and $\mu>0$ the radius of a disc with center at $q$. Then $W$ is zero for $z$ outside the disc $D_{\mu}^{2}$ and $W$ is equal $v$ for $z$ inside $D_{\mu / 2}^{2}$. Moreover $W \rightarrow 0$ when $v \rightarrow 0$. Define the diffeomorphism $f: \mathbf{R}^{2} \rightarrow \mathbf{R}^{2}$ for given $v$ and $\mu$ by $f(z ; q, v, \mu)=\varphi_{1}(z ; W(\cdot, q, v, \mu))$, where $\varphi_{1}$ is the time one diffeomorphism of the $W$-flow. Since $W \rightarrow 0$ when $v \rightarrow 0, f$ is near the identity inside $\Gamma_{\mu / 2}^{2}$ for small $v$ and $f$ is the identity outside $D_{\mu}^{2}$ because $W$ is zero there. Since $f(q)=q+v$, taking $v=p-q$ gives $f$ all properties required in the proof of (4.9).

REMARK 3. If the singularities of $X$ are hyperbolic, we have the Closing Lemma. In fact, by Remark 1 we can get a nonsingular box-chain through $p$, directly from the Box-chain Lemma and make it simple by (4.5). By the same arguments used in the proof of (4.9), we can get a closed orbit through $p$ for a vector field arbitrarily close to $X$.

We now remove the singularities of the closed curve given by the Singular Closing Lemma to obtain our main result.

Closing LEMma. Suppose the singularities of $X \in \mathscr{X}^{r}\left(\mathbf{R}^{2}\right)$ are all semihyperbolic or satisfy the shadow property. Given a prolongationally recurrent point $p \in R(X)$, and a neighborhood $\mathscr{U}$ of $X$ in $\mathscr{X}^{r}\left(\mathbf{R}^{2}\right)$, there exists $Z \in \mathscr{U}$ with a closed orbit through $p$.

ProOF. We choose a smaller neighborhood $\mathscr{U}^{\prime} \subset \mathscr{U}$ of $X$, and by the Singular Closing Lemma there exists a vector field $Y \in \mathscr{U}^{\prime}$ such that $Y$ has a closed curve $\lambda$ through $p$, composed of trajectory arcs and singularities $p_{i}$ satisfying the shadow property (1.7) and semihyperbolic singularities $q_{j}, i=1, \ldots, m, j=1, \ldots, n$. We consider a neighborhood $\mathscr{U}_{0}$ of $Y, \mathscr{U}_{0} \subset \mathscr{U}$.

Since each $p_{i}$ satisfies the shadow property, by (1.7) there exists a neighborhood $W_{i}$ of $p_{i}$ such that every $(\varepsilon, 1)$-chain in $W_{i}$ can be $\delta$-shadowed by a single trajectory arc and $\delta \rightarrow 0$ uniformly as $\varepsilon \rightarrow 0$. Thus we can have a $(\varepsilon, 1)$-chain $\left[a_{i}, b_{i}\right]$, $\left[c_{i}, d_{i}\right]$ with the two trajectory arcs of $\lambda$ contained in $\bar{W}_{i}$, which are respectively the trajectory arc that goes to $p_{i}$ and the trajectory arc that leaves $p_{i}$. We take $a_{i}$, 


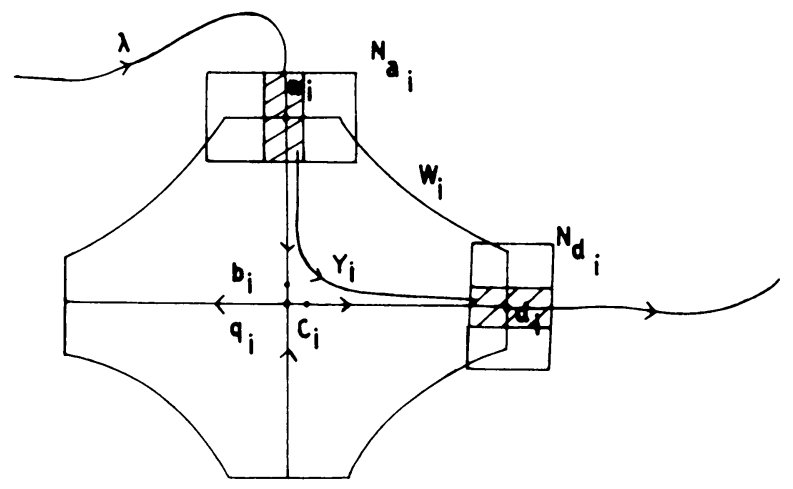

FigURE (4.8)

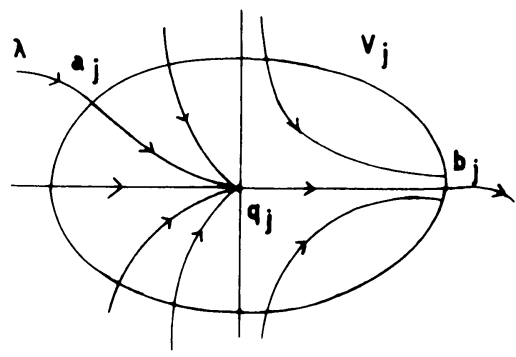

(a)

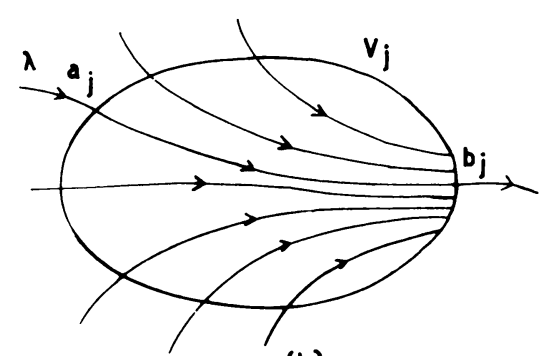

(b)

FiguRE (4.9)

$d_{i}$ as the intersection points of $\lambda$ with $\partial W_{i}$. By the shadow property, this chain is $\delta$-shadowed by a single trajectory arc $\gamma_{i}$ of $Y$. See Figure (4.8).

Consider disjoint flowboxes $N_{a_{i}}$ and $N_{d_{i}}$ around $a_{i}$ and $d_{i}$, for each $i$. By (3.5) we have a $c$-central portion in $N_{a_{i}}$ and in $N_{d_{i}}$ such that any two points in opposite transversal edges of the $c$-central portion of $N_{a_{i}}$ and of $N_{d_{i}}$ can be connected by a trajectory arc of $Z_{i}$ and of $Z_{i}^{\prime}, Z_{i}, Z_{i}^{\prime} \in \mathscr{U}_{0}$, with $Z_{i} \equiv Y$ and $Z_{i}^{\prime} \equiv Y$ outside $N_{a_{i}}$ and $N_{d_{i}}$ respectively. Since $\delta \rightarrow 0$, uniformly as $\varepsilon \rightarrow 0$, we can have the trajectory arc $\gamma_{i}$ leaving $\partial N_{a_{i}}$ and arriving at $\partial N_{d_{i}}$ in their $c$-central portions. Next we connect $\lambda$ to $\gamma_{i}$ by a $Z_{i}$-trajectory across $N_{a_{i}}$ and $\gamma_{i}$ to $\lambda$ by a $Z_{i}^{\prime}$-trajectory across $N_{d_{i}}$. Thus we remove $p_{i}$ from $\lambda$, for each $i$.

Clearly the semihyperbolic singularities of $Y$ that could belong to the box-chain loop $\Gamma^{\prime}$ which starts the closed curve $\lambda$ are saddle-nodes and weak saddles. The weak saddles satisfy the shadow property and they are removed as above. Then the singularities $q_{j}$ to be removed are all saddle-nodes.

Choose a small neighborhood $V_{j}$ of each $q_{j}$ such that the only singularity of $Y$ in $V_{j}$ is $q_{j}$, while $V_{1}, \ldots, V_{n}, N_{a_{1}}, \ldots, N_{a_{n}}, N_{d_{1}}, \ldots, N_{d_{n}}$ are disjoint and in $V_{j}$ we can write

$$
Y=f\left(x_{1}, x_{2}\right) \frac{\partial}{\partial x_{1}}+g\left(x_{1}, x_{2}\right) \frac{\partial}{\partial x_{2}},
$$

where the saddle-node $q_{j}(0,0)$. By the remarks following $(1.8)$ we have $g\left(x_{1}, 0\right) \equiv 0$ and $f\left(x_{1}, 0\right)>0$ or $f\left(x_{1}, 0\right)<0$ for $x_{1} \neq 0$. We assume $f\left(x_{1}, 0\right)>0$. The closed 
curve $\lambda$ comes in $V_{j}$ by a trajectory arc crossing the boundary $\partial V_{j}$ at $a_{j}$ and leaves $V_{j}$ by the unstable separatrix at $b_{j}$. (See Figure (4.9)(a).)

Suppose $\varphi_{j}$ is a $C^{\infty}$ function with $0<\varphi_{j}\left(x_{1}, x_{2}\right) \leq 1$ inside $V_{j}$ and $\varphi_{j}\left(x_{1}, x_{2}\right) \equiv$ 0 outside $V_{j}$. Then for arbitrarily small values of $\varepsilon_{j}>0$ and of appropriately chosen $\mu_{j}$, the family

$$
Y_{j}=Y+\varepsilon_{j} \varphi_{j} \frac{\partial}{\partial x_{1}}+\mu_{j} \varphi_{j} \frac{\partial}{\partial x_{2}}
$$

has the following properties, based on the continuity of integral curves with respect to parameters.

(1) $Y_{j} \in \mathscr{U}_{0}$

(2) $Y_{j} \equiv Y$ off $V_{j}$

(3) $q_{j}$ is removed and $Y_{j}\left(x_{1}, x_{2}\right) \neq 0$ for $\left(x_{1}, x_{2}\right) \in V_{j}$,

(4) the trajectory arc of $Y_{j}$ through $a_{j}$ passes through $b_{j}$. (See Figure (4.9)(b).)

Since $N_{a_{1}}, N_{a_{2}}, \ldots, N_{a_{m}}, N_{d_{1}}, N_{d_{2}}, \ldots, N_{d_{m}}, V_{1}, V_{2}, \ldots, V_{n}$ are disjoint and $\operatorname{supp}\left(Z_{i}-Y\right)=N_{a_{i}}, \operatorname{supp}\left(Z_{i}^{\prime}-Y\right)=N_{d_{i}}$, and $\operatorname{supp}\left(Y_{j}-Y\right)=V_{j},(4.8)$ shows that $Z=Y+\sum\left(Z_{i}-Y\right)+\sum\left(Z_{i}^{\prime}-Y\right)+\sum\left(Y_{j}-Y\right), \quad i=1, \ldots, m, j=1, \ldots, n$, lies in $\mathscr{U}_{0} \subset \mathscr{U}$ and by construction $Z$ has a closed orbit through $p$.

ACKNOWLEDGment. The author is grateful to Charles Pugh for helpful suggestions. Thanks are also due to Cesar Camacho for productive comments. I also wish to thank the University of California at Berkeley for kind hospitality during the preparation of this paper.

\section{REFERENCES}

1. J. Auslander, Generalized recurrence in dynamical systems, Contributions to Differential Equations 3 (1964), 65-74.

2. N. P. Bhatia and G. P. Szegö, Stability theory of dynamical systems, Springer-Verlag, Berlin and New York, 1970.

3. F. Dumortier, Singularities of vector fields on the plane, J. Differential Equations 23 (1977), 53-106.

4. C. Gutierrez, On the $C^{r}$-closing lemma for flows on the torus $T^{2}$, Ergodic Theory and Dynamical Systems (to appear).

5., A counterexample to a $C^{2}$ closing lemma (to appear).

6. R. Mañe, An ergodic closing lemma, Ann. of Math. (2) 116 (1982), 503-541.

7. P. Mendes, On stability of dynamical systems on open manifolds, J. Differential Equations 16 (1974), 144-167.

8. Z. Nitecki, J. Kotus and M. Krych, Global structural stability of flows on open surfaces, Mem. Amer. Math. Soc. (to appear).

9. Z. Nitecki, Explosions in completely unstable flows. I, Preventing explosions, Trans. Amer. Math. Soc. 245 (1972), 43-61.

10. __ Explosions in completely unstable flows. II, Some examples, Trans. Amer. Math. Soc. 245 (1978), 63-88.

11. J. Palis and W. de Melo, Geometric theory of dynamical systems, Springer-Verlag, Berlin and New York, 1983.

12. M. M. Peixoto, Structural stability on two dimensional manifolds, Topology 1 (1962), 101-120.

13. __ On an approximation theorem of Kupka and Smale, J. Differential Equations 3 (1967), 214-227.

14. M. M. Peixoto and C. C Pugh, Structural stability on open manifolds in never dense, Ann. of Math. (2) 87 (1968), 423-430.

15. C. C. Pugh, The closing lemma, Ann. of Math. (2) 89 (1967), 956-1009.

16. __ An improved closing lemma and a general theorem, Amer. J. Math. 89 (1967), 1010-1021. 
17. __ Against the $C^{2}$ closing lemma, J. Differential Equations 17 (1975), 435-443.

18. C. C. Pugh and C. Robinson, The $C^{1}$ closing including Hamiltonians, Ergodic Theory and Dynamical Systems 3 (1983), 261-313.

19. C. C. Pugh, The $C^{1}$-connecting lemma, a counterexample (preprint).

20. C. C. Pugh and M. L. A. Peixoto, The planar closing lemma for chain recurrence (to appear).

21. J. Sotomayor, Generic one-parameter families of vector fields on two-dimensional manifolds, IHES, Extrait de Publications Mathematiques, 43.

22. T. Ura, Sur les courbes définies par les équations différentielles dans l'espace à $m$ dimensions, Ann. Sci. École Norm. Sup. Pisa (3) 70 (1953), 287.

23. __ Sur le courant exterieur à une région invariante, Funkcial. Ekvac. 2 (1958), 143-200.

Escola Nacional de CiênCias Estatísticas-ENCE, RUa AndRÉ CaValCanti, 106, 20321 RIO DE JANEIRO-RJ-BRASIL 